\title{
Septum Magnet MNP-23 for the CERN PS Experimental Area and Its Fast Interlock System
}

\author{
J. Borburgh, T. Masson, A. Prost, and T. Zickler
}

\begin{abstract}
Two MNP-23 septum-like magnets are installed at CERN in the transfer line from the Proton Synchrotron (PS) to the East Hall Experimental Area. They are exposed to extremely high doses of ionizing radiation. In the past, the magnets experienced two catastrophic failures due to overheating of its coils and cannot be repaired. The magnets of improved design which is subject of this article are built as replacements for the magnets presently installed.

The MNP-23 is a resistive $\mathrm{C}$ shaped iron-dominated magnet made of solid low carbon steel blocks. The excitation windings consist of two water-cooled coils wound from hollow copper conductor. The septum design of these magnets implies a high current density which requires an efficient water cooling system. The newly designed cooling circuit provides better cooling performance and more reliability.

To avoid failures due to coil overheating, an elaborate interlock system was developed and installed. It consists of two parts: firstly a slow, more classic sensor, to detect gradual water blockages and inlet cooling water temperature rises and secondly a fast response device to detect sudden loss of water cooling due to external leaks or sudden blockage of one of the water cooling circuits in the coil. The article outlines the unusual and challenging design of the magnet and its interlock system and describes the hardware as well as its performance.
\end{abstract}

Index Terms-Accelerator magnets, control equipment, cooling, particle beam transport.

\section{INTRODUCTION}

$\mathbf{T}$ HE transfer-line from the PS to the East Hall Experimental Area provides proton beams of different energies (from $3.5 \mathrm{GeV} / \mathrm{c}$ up to $24 \mathrm{GeV} / \mathrm{c}$ ) to various experiments, mainly for the new LHC detector developments. Two MNP-23 septum-like magnets are actually installed in this transfer line.

One of them is placed in the slow extraction line from the PS to the Experimental Area, the other one is located directly downstream the target area of the Experimental Zone in the East Hall. Particularly the latter one is exposed to extremely high doses of ionizing radiation. Therefore normal maintenance is prohibited and any intervention in this area is rather difficult. Two magnets have already failed in the past and cannot be repaired. Due to the lack of maintenance, the high current density, the high-pressure water-cooling and the high radiation level, the undisturbed and reliable performance of the old magnets could not be guaranteed for the future. Shortly before the arrival of the first newly fabricated magnet, a leaking coil of the MNP-23 installed in the

Manuscript received October 20, 2003.

The authors are with the CERN, CH-1211 Geneva 23, Switzerland (e-mail: thomas.zickler@cern.ch).

Digital Object Identifier 10.1109/TASC.2004.829984
TABLE I

MNP-23 MAGNET PARAMETERS

\begin{tabular}{lll}
\hline \hline \multicolumn{1}{c}{ Parameter } & \multicolumn{1}{c}{ Value } & Unit \\
\hline Flux density in gap & 1.41 & $\mathrm{~T}$ \\
Yoke gap height & 80 & $\mathrm{~mm}$ \\
Magnetic gap height (vacuum chamber installed) & 60 & $\mathrm{~mm}$ \\
Yoke length & 2500 & $\mathrm{~mm}$ \\
Total number of turns & $2 \times 25$ & \\
Number of cooling circuits & 50 & \\
Nominal current & 1350 & $\mathrm{~A}$ \\
Nominal voltage & 350 & $\mathrm{~V}$ \\
Nominal power dissipation & 471 & $\mathrm{~kW}$ \\
Electrical resistance at 20 ${ }^{\circ} \mathrm{C}$ & 217 & $\mathrm{~m} \Omega$ \\
Cooling water pressure drop & 18 & $\mathrm{bar}$ \\
Cooling water inlet pressure interlock & 23 & $\mathrm{bar}$ \\
Velocity of cooling water on septum side & 10.2 & $\mathrm{~m} / \mathrm{s}$ \\
Cooling water flow per magnet & $2 \times 108$ & $1 / \mathrm{min}$. \\
Cooling water flow interlock & $2 \times 105$ & $1 / \mathrm{min}$. \\
Cooling water temperature rise (nominal) & 31 & ${ }^{\circ} \mathrm{C}$ \\
Temperature interlock on cooling water outlet & 65 & ${ }^{\circ} \mathrm{C}$ \\
Average copper temperature interlock (average & 50 & ${ }^{\circ} \mathrm{C}$ \\
temperature of a layer of 5 turns) & & \\
Thermostat switch-off temperature (on every & 65 & ${ }^{\circ} \mathrm{C}$ \\
winding) & & \\
\hline \hline
\end{tabular}

slow extraction line caused a six weeks physics stop in the south branch of the Experimental Area.

\section{MAGNET DESIGN AND CONSTRUCTION}

\section{A. Design Requirements}

Since the new magnets have to replace the existing ones in their function, most of their main parameters, in particular the overall outer dimensions had to be taken over from the previous design. The limited space in the tunnel and the proximity of the adjacent transfer line branch imposed the septum-like design. The maximum required bending angle is $43 \mathrm{mrad}$ for the $24 \mathrm{GeV} / \mathrm{c}$ proton beam respectively $72 \mathrm{mrad}$ for the $15 \mathrm{GeV} / \mathrm{c}$ beam, which requires in total more than 67100 ampere-turns. A list of the main design parameters is given in Table I.

\section{B. Yoke Construction}

The dipoles are iron-dominated magnets. The $2500 \mathrm{~mm}$ long magnetic circuit consists of two identical pole blocks made of solid low carbon steel and an intermediate piece of the same material, very accurately positioned and held together by tie-bolts. A retaining plate is fixed on the front side. This plate acts as shield to keep away the fringe field from the adjacent beam line and to fix the coil in its proper position. To make the most use of the magnet gap height and to increase the beam aperture, a special rectangular vacuum chamber is incorporated in the magnet. 


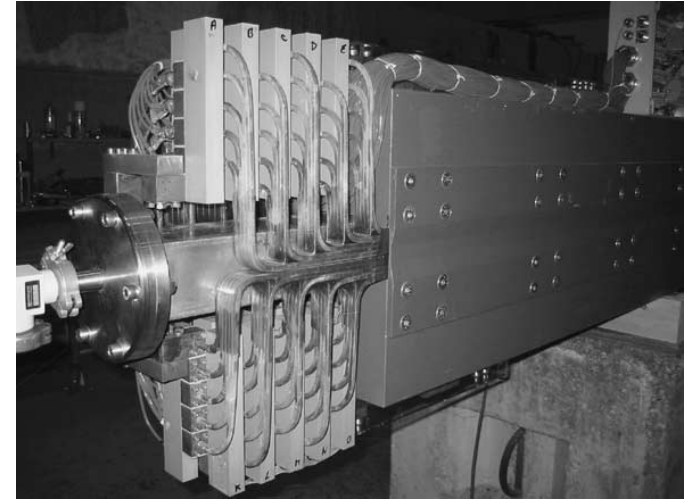

Fig. 1. The connection side of the MNP-23 Septum Magnet. The KETRON PEEK Insulators, the septum side coil ends, the thermal switch cables, and the rectangular vacuum chamber are already installed. The stainless steel water pipes and the fast interlock pick-up wires are not shown on this picture.

It is made of two horizontal, $10 \mathrm{~mm}$ thick magnetic steel plates and two thin vertical nonmagnetic stainless steel plates welded together. Because the field quality is determined by the horizontal plates, they have to be precisely machined and aligned perfectly parallel with respect to each other.

\section{Excitation Coil and Cooling System}

The excitation winding consists of two water-cooled coils. The coils are wound from hollow oxygen-free copper conductor and insulated by glass tapes and vacuum impregnated by epoxy resin. Each coil comprises five layers of five turns. The two coils are electrically connected in series. The septum like design of these magnets causes a very high current density on the septum side of the coil (up to $77 \mathrm{~A} / \mathrm{mm}^{2}$ ) which requires a very efficient water cooling system. In fact, the cooling system has to deal with more than $470 \mathrm{~kW}$ dissipated power.

The newly designed cooling circuit (see Fig. 1) provides better cooling performance and more reliability. It was very important to optimize the dimensions of the conductor cross-sections, the water pressure and the number of cooling circuits to guarantee a sufficient cooling performance in order to avoid a heating of the coil beyond $65^{\circ} \mathrm{C}$ and to ensure that the velocity of the cooling water does not exceed the critical limit of $10 \mathrm{~m} / \mathrm{s}$ too far in order to minimize cavitation effects in the cooling ducts. Therefore, each turn consists of one length of "septum conductor" and one length of "return conductor." They are connected by brazing and by means of copper sleeves with tapped holes on the coil heads. On the septum side of the coil, a square cross-section of $5 \mathrm{~mm} \times 5 \mathrm{~mm}(3 \mathrm{~mm}$ circular cooling duct) was used to keep the outer dimensions of the coil as small as possible. The "return conductor" with a rectangular cross-section $(12 \mathrm{~mm} \times 4.9 \mathrm{~mm}$ with a rectangular cooling duct of $9.5 \mathrm{~mm} \times 2.4 \mathrm{~mm}$ ) has been selected to reduce current density, the temperature increase, and the cooling water velocity.

The 50 individual hydraulic circuits (one per turn) are connected in parallel to the insulating water collector. The insulating water collectors are made of KETRON PEEK. This material has been chosen since it combines the attributes of sufficient radiation hardness and good electrical insulation properties. In addition, the excellent mechanical strength of this mate- rial makes machining easy and reinforcement inserts like "helicoils" obsolete.

To obtain a continuous high quality of the 200 brazed joints per magnet, special brazing tools have been developed.

Stainless steel $\left(1 / 2^{\prime \prime}\right)$ tubes and stainless steel ferrule fittings connect the insulating water collectors to the main water manifolds.

\section{THE INTERLOCK SYSTEM}

The interlock system is designed to fulfill three tasks: a) surveillance of the magnet coil cooling and temperature; $b$ ) protection of the magnet in case a sudden loss of cooling occurs in one or more cooling circuits; and c) monitoring of the magnet thermal state in general.

The surveillance of the magnet coil temperature and cooling is done with a hard wired electronics system (see Fig. 2) with 'classical' sensors and a solenoid valve at the high pressure side of the cooling circuit. This system can detect a gradual heating of the magnet coils, and it also monitors and controls the magnet cooling. As soon as one of the set limits is exceeded, this system shuts down the magnet power supply.

The protection of the magnet from a sudden loss in cooling in one of the cooling circuits is done with a software based, so called 'fast interlock' system, which feeds into the hard wired classic interlock electronics rack. This fast protection system prevents the magnet from overheating when only one cooling circuit gets blocked because the temperature of the corresponding turn would rise very quickly, damaging the magnet coil permanently. Classic thermostats are too slow for this application, and the flow meter is too inaccurate to detect such a small drop in water flow, compared to the total water flow of the magnet. This system has to react within $20 \mathrm{~ms}$ between the moment of the coil turn overheating and the shut down of the power supply.

Finally this PC based 'fast protection' system is also used to display the present state of the magnet, showing on a screen the status of all interlocks (with the failures memorized since the last reset), as well as the main parameters of the magnet such as current, actual power dissipation and cooling water flow and temperature. This allows the user to have in one place all relevant data of the magnet to be retrieved in case of a magnet failure.

The following paragraphs describe the strategic choices made to design the interlock system, and the logic used to protect the magnet. A more detailed description of the system at hardware level is given in [1].

\section{A. The Classic Hard Wired Interlock System}

The classic interlock system is a hard wired system that groups all passive sensors to protect the magnet from being powered while overheating, for example due to slowly blocking of the cooling circuits by corrosion, or variation of the cooling water pressure or temperature at the inlet. The interlock system feeds into the power converter the 'magnet fault' interlock. If this interlock is opened by the magnet interlock system the power supply is immediately shut down. The magnet interlock system also monitors and controls the cooling water of the 


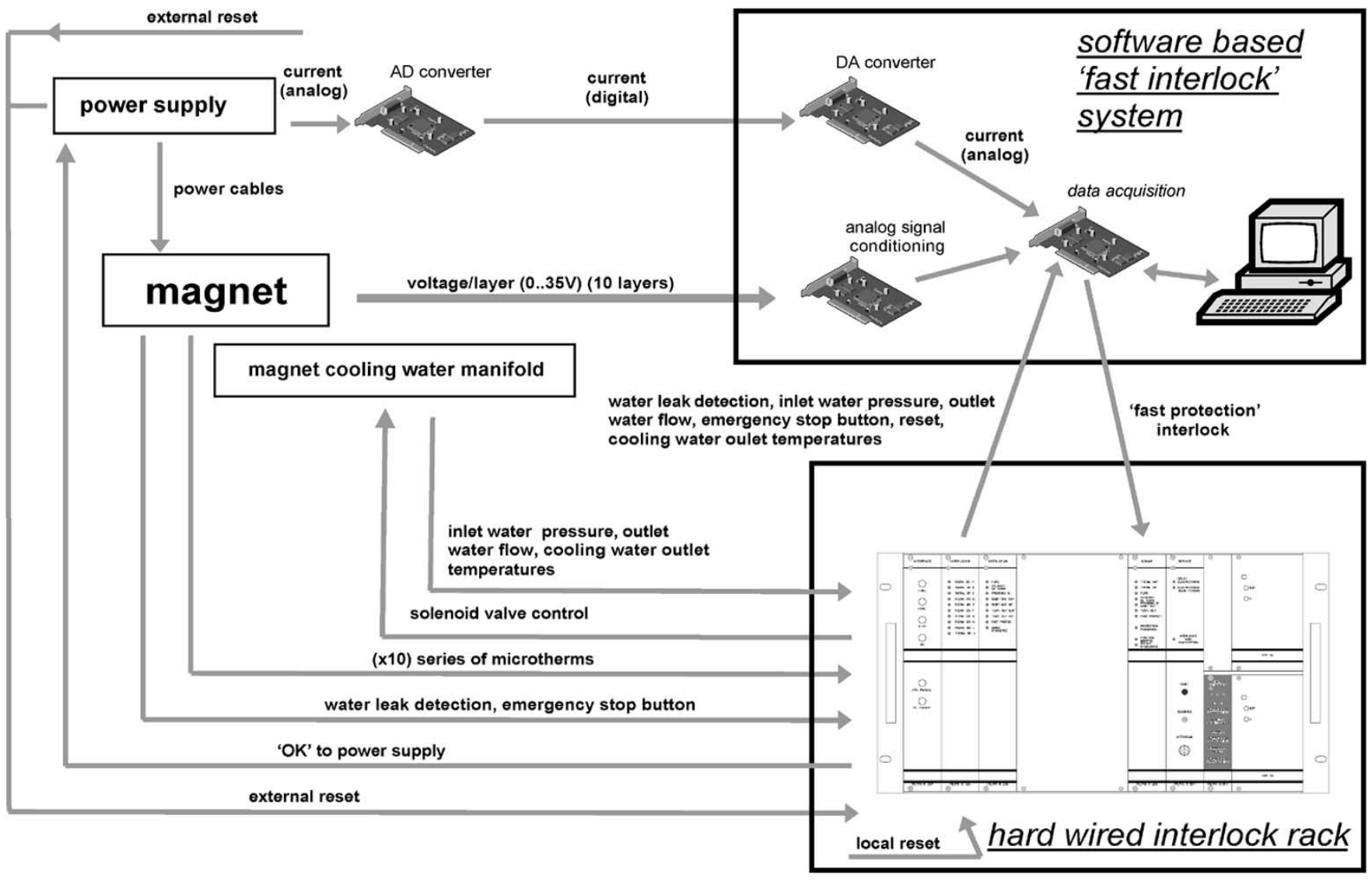

Fig. 2. The principle of the interlock and surveillance system for the MNP23 type magnets. A software based 'fast interlock' system forms as an additional input for the classic hard wired interlock system. The classic interlock system groups all interlock sensors, can shut down the power supply if necessary and switch on and off the magnet water flow.

magnet through a solenoid valve mounted on the high pressure cooling water manifold, which can be either fully opened or closed.

The system shuts down the power supply and maintains the cooling if it detects overheating of the magnet through either the cooling water outlet temperature limit or the thermostats glued on every coil winding.

The system shuts down the power supply and the cooling system when the cooling water inlet pressure or water flow are too low, when a leak is detected on the water retaining covers by a float switch under the hydraulic coil connections, when the fast interlock is activated or when the 'Personnel safety switch' is pressed.

The values for all different interlocks levels of this classic magnet interlock system are mentioned in Table I. The system uses mostly standard electronics cards as for all other septa magnets of the CERN Complex, to reduce the variety of spares to be made. In the event of a sensor failure, the interlock sensors input card allows easy deactivation of each or all sensors by turning around a printed circuit board, if the sensor can be considered as redundant. In this case however, deactivation of a sensor is indicated by the illumination of a front panel led, which indicates that the system has been reconfigured. One such case can occur at start up of the magnet when the leak detection float switch may be inadvertently activated by the high stray magnetic field. For the time being this interlock channel is deactivated.

\section{B. The 'Fast Interlock' System}

The fast interlock system calculates the average temperature of the copper conductor per layer of 5 coil turns, and also detects any possible short circuits between coil turns. If either the preset temperature or an inter-turn short circuit is detected, it opens the interlock that feeds into the classic interlock system. The system hardware is an industrial PC running Windows and a National Instruments DAQ 6023E data acquisition board. An application developed with National Instruments Labview takes care of the data processing. This choice was the most economical within the infrastructure already present at CERN.

To limit the hardware, the system is based (see Fig. 2) on a voltage drop measurement across each layer of 5 coil turns instead of individual turns, combined with a measurement of the current through the magnet. The 10 resulting voltages are sent by means of a $7 \mathrm{kV}$ rated, screened, twisted pair cable to the interlock system rack, approximately 150 meters from the magnet. This $0-35 \mathrm{~V}$ signal is conditioned to a normalized 0-10 V signal that is sampled by the 12 bit data acquisition board. The current is digitized at the power supply to reduce the noise picked up between the power supply and the interlock rack, before being sent to the interlock rack. There it is converted into a normalized 0-10 V signal as well, so that signal can be treated as the other 10 signals by the data acquisition board in the PC. An additional input channel available on the board can be used to 
measure the $\mathrm{dI} / \mathrm{dt}$, but this feature is presently unused to reduce cost and complexity.

The average copper temperature per layer is derived from an on-line electrical resistance measurement, using the acquired data as follows:

$$
R_{\text {layer }}=\frac{U_{\text {layer }}}{I}-\frac{L_{\text {layer }} \frac{d I}{d t}}{I}
$$

In our case of a DC magnet it is assured that

$$
\frac{d I}{d t}=0
$$

To make sure this remains valid during magnet power up, the power supply maximum ramp up speed is set to $250 \mathrm{~A} / \mathrm{s}$. The average temperature can then be calculated using

$$
T_{\text {layer }}=T_{0}+\left[\frac{R_{\text {layer }}-R_{\text {layer } 0}}{\alpha \cdot R_{\text {layer } 0}}\right]
$$

where $\alpha$ is equal to the electrical temperature coefficient of copper, and $\mathrm{T}_{0}$ the room temperature at which the cold resistance measurements of each layer $\mathrm{R}_{\text {layer0 }}$ were taken. Once any of the average layer temperatures exceeds the set limit (see Table I), the fast interlock is triggered, and the graphical interface displays which layer triggered the interlock.

The system also detects inter-turn short circuits by comparing the voltage drops over each layer. The program normalizes these voltage drops with respect to the corresponding layer resistance $\mathrm{R}_{\text {layero }}$. A short circuit between turns within one layer is characterized by a $20 \%$ reduction of the normalized layer voltage drop. An inter-layer short circuit may show only a $10 \%$ reduction of the normalized layer voltage drop. By comparison of all normalized layer voltages, the program detects the inter-turn short circuits. In the event of a short circuit, the fast interlock trigger is activated along with an onscreen presentation indicating the layer at fault.

In fact at these currents the power dissipation is so low, the risk of overheating of a coil turn, and subsequent damage are virtually nil. To reduce the risk of faulty interlock triggers during power up of the magnet, the fast interlock system is inactive for currents below $250 \mathrm{~A}$.

To make sure the whole process is completed within $20 \mathrm{~ms}$ as stipulated in the design specifications, the graphical interface had to be reduced to a more basic output format. By doing so, the present set-up manages a full cycle within $17 \mathrm{~ms}$. In case the program has to manage two magnets simultaneously, an optimized strategy has already been developed to complete the full cycle for both within the requested $20 \mathrm{~ms}$ reaction time.

\section{The Monitoring Screen}

To help diagnose the status of the magnet for maintenance purposes or in case of failures, all data taken into account by the classic system are transferred from the classic interlock system to the $\mathrm{PC}$ via the data acquisition board. These data are displayed by the graphical user interface next to the information of the fast interlock system itself, without further treatment. Additionally an approximation of the actual power dissipation in the magnet is displayed, based on the current and voltage measurements on the coil. Although some additional process time is used for all this, it is very useful for operation and it does not prevent the entire process cycle to be completed within the set limits.

\section{CONCLUSION}

The magnet and its dedicated protection and control system have now been operational for almost two years. No problems have occurred so far. To further improve the reliability of the system it is suggested to power the PC based 'fast interlock' system from an un-interruptible power supply, which is available near the electronics racks, to avoid long start ups and risks of damage after a power cut. The present float switches should replaced by switches resistant to magnetic fields, to make the leak water detection operational.

\section{REFERENCES}

[1] T. Masson and A. Prost, "Surveillance et protection des aimants type MNP23,” CERN, AB-Note-2003-039 BT, June 2003. 